9-2012

\title{
A Clinically Significant Interaction Between Tacrolimus and Multiple Proton Pump Inhibitors in a Kidney Transplant Recipient
}

Michelle Maguire

Thaddeus T. Franz

CedarvilleUniversity, tfranz@cedarville.edu

David S. Hains

Follow this and additional works at: https://digitalcommons.cedarville.edu/

pharmacy_practice_publications

Part of the Pediatrics Commons, Pharmacy and Pharmaceutical Sciences Commons, and the Reproductive and Urinary Physiology Commons

\section{Recommended Citation}

Maguire, Michelle; Franz, Thaddeus T.; and Hains, David S., "A Clinically Significant Interaction Between Tacrolimus and Multiple Proton Pump Inhibitors in a Kidney Transplant Recipient" (2012). Pharmacy Practice Faculty Publications. 95.

https://digitalcommons.cedarville.edu/pharmacy_practice_publications/95 


\section{Pediatric Transplantation \\ Omatial Journat of the Internamonat. Pediatric Transplant Associamon

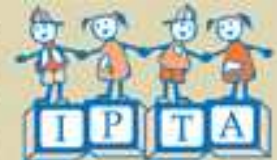

\section{A Clinically Significant Interaction between Tacrolimus and Multiple Proton Pump Inhibitors in a Kidney Transplant Recipient}

\begin{tabular}{|r|l|}
\hline Journal: & Pediatric Transplantation \\
\hline Manuscript ID: & PEDTRANS-11-CR-0096.R1 \\
\hline Manuscript Type: & Case Report \\
\hline $\begin{array}{r}\text { Date Submitted by the } \\
\text { Author: }\end{array}$ & $\mathrm{n} / \mathrm{a}$ \\
\hline Complete List of Authors: & Maguire, Michelle \\
\hline Keywords: & $\begin{array}{l}\text { tacrolimus, proton pump inhibitors, drug interaction, pediatric renal } \\
\text { transplantation, CYP 2C19, pharmacogenetics }\end{array}$ \\
\hline
\end{tabular}

\section{SCHOLARONE ${ }^{\text {TM }}$ \\ Manuscripts}




\title{
A Clinically Significant Interaction between Tacrolimus and Multiple Proton Pump Inhibitors in a Kidney Transplant Recipient
}

\author{
Michelle Maguire ${ }^{1}$, Thaddeus Franz PharmD², and David S. Hains, M.D.,4 \\ ${ }^{1}$ College of Pharmacy, The Ohio State University, ${ }^{2}$ Department of Pharmacy, \\ Nationwide Children's Hospital, ${ }^{3}$ Center for Clinical and Translational Research, The \\ Research Institute at Nationwide Children's Hospital, 4 Division of Pediatric \\ Nephrology, Nationwide Children's Hospital, Columbus, Ohio.
}

Credited Institution: Division of Pediatric Nephrology, Nationwide Children's Hospital, Columbus, Ohio

\section{Corresponding Author:}

David S. Hains, M.D.

700 Children's Drive

Columbus, OH 43206

David.Hains@nationwidechildrens.org

Telephone: (614) 722-2683

Fax: (614) 722-6482 


\begin{abstract}
:
The shared metabolism of proton pump inhibitors (PPIs) and tacrolimus through the cytochrome p450 (CYP) enzyme system has been associated with clinically significant drug interactions, especially in patients who are classified as CYP 2C19 poor metabolizers. However, existing data is conflicting, indicating that a single mechanism does not account for all interactions. A drug interaction between tacrolimus and omeprazole, esomeprazole, but not lansoprazole, occurred in an 18year-old female kidney transplant recipient classified as a CYP 2C19 extensive (normal) metabolizer. This case suggests further research is needed to establish the definitive mechanism of this potentially serious drug-drug interaction. Physicians prescribing PPIs in organ transplant recipients with tacrolimus immunosuppression should consider close pharmacokinetic monitoring of tacrolimus when starting or switching a PPI.
\end{abstract}




\section{Background:}

Tacrolimus is a highly effective immunosuppressant drug used in combination to prevent solid organ transplant rejection. However, the narrow therapeutic window between under-dosing predisposing to increased risk of organ rejection and over-dosing leading to side effects such as acute and chronic nephrotoxicity, renal vasoconstriction and myelosuppression necessitates close monitoring of blood concentrations. Tacrolimus is absorbed through the intestinal multi-drug efflux transporter p-glycoprotein (PgP) and metabolized via hepatic CYP 3A enzyme systems, making it susceptible to many clinically significant drug interactions [1]. Most interactions are well known and managed through increased monitoring of tacrolimus blood concentrations, but few are as poorly elucidated interactions as with the proton pump inhibitors (PPIs).

Hepatic CYP 2C19 and 3A4 metabolize PPIs, and each PPI is metabolized differentially through the two pathways [2]. The shared hepatic CYP 3A4 system with tacrolimus is the presumed source of the interaction, but the exact pathway remains controversial. Many researchers have shown correlations between interaction potential of PPIs with tacrolimus and pharmacogenetic variability of CYP 2C19. CYP 2C19 genotypes classify patients as extensive metabolizers (EMs), intermediate metabolizers (IMs), or poor metabolizers (PMs) [3]. Poor metabolizers have markedly reduced activity of the 2C19 enzyme, leading to increased reliance on 3A4 for PPI metabolism [3]. Inhibition of tacrolimus clearance subsequently leads to supratherapeutic tacrolimus levels. However, conflicting data has spurred research into other pathways of the interaction, including intestinal PgP and hepatic CYP 3A5 genetic variability, all with more inconclusive or negative results [4-6].

In this case report, we present a renal transplant patient with a clinically relevant interaction between tacrolimus and esomeprazole, omeprazole, but not lansoprazole.

\section{Case Report:}

An 18-year-old Caucasian female with Wegener's Granulomatosis underwent a living donor renal transplant and placed on tacrolimus and mycophenolate for immunosuppression as well as lansoprazole for acid blockade. Concomitant chronic transplant medications included prednisone, sulfamethoxazole/trimethoprim, valganciclovir, and carvedilol. Tacrolimus blood levels were monitored routinely posttransplantation, with a goal range of trough concentrations between 5-8 ng/mL for maintenance immunosuppression. The patient maintained blood levels in this target range with $6 \mathrm{mg} /$ day of tacrolimus in two divided doses. Eight months post-transplant, lansoprazole (30mg/day) was switched to esomeprazole (40mg/day) due to insurance formulary changes. 
The patient presented one month later with fatigue and persistent sinusitis unresolved with courses of antibiotics. Tacrolimus blood levels were found to be elevated at $27.4 \mathrm{ng} / \mathrm{mL}$. The patient reported receiving a 10-day course of azithromycin from an outside physician, which she completed over 2 weeks prior to the first elevated tacrolimus trough, followed by a 10-day course of cefdinir completed 3 days prior to first elevated tacrolimus trough. The patient confirmed adherence to her tacrolimus regimen, and denied use of over-the-counter medications, grapefruit juice, and illicit drugs or alcohol. Her previous 9 months of tacrolimus levels were within the therapeutic window each time. Liver function tests of AST and ALT were within normal limits, and urine drug and toxicology screens were negative. The antibiotics were not considered likely causes of the elevated tacrolimus levels due to the time course of symptomatology and presentation in relation to antibiotic cessation. The tacrolimus dose was held for 24 hours and then reduced to $4 \mathrm{mg} /$ day. The blood levels were re-drawn the next morning and decreased to $9.6 \mathrm{ng} / \mathrm{mL}$.

Ten days later, the patient was admitted to the hospital with elevated serum creatinine and a tacrolimus level of $13.7 \mathrm{ng} / \mathrm{mL}$. She reported nausea and vomiting for several days, but reported no missed doses of tacrolimus. A second 21-day course of cefdinir had been prescribed prior to this presentation and was not considered as a causative agent due to the time course of symptomatology and lack of established evidence for an interaction with tacrolimus metabolism. Cefdinir was thus continued through and beyond the hospital admission. The tacrolimus was withdrawn and the patient was switched to omeprazole $40 \mathrm{mg}$ /day as per hospital formulary. Tacrolimus levels continued to remain elevated up to $20.1 \mathrm{ng} / \mathrm{mL}$. Figure 1 shows the course of tacrolimus blood concentrations and dosage during this time.

Physician and pharmacist review of the medication profile revealed a potential drug interaction between esomeprazole, omeprazole and tacrolimus. The patient was placed back on the lansoprazole. Tacrolimus levels normalized and symptoms of nausea and vomiting resolved. Within one week, the target range of $5-8 \mathrm{ng} / \mathrm{mL}$ was reached and tacrolimus titrated back up to the initial dose of $6 \mathrm{mg} /$ day. After obtaining written informed consent, a blood sample was sent to an outside facility for a cytochrome P450 2C19 genetic test done by polymerase chain reaction followed by DNA sequence analysis. The patients genotype came back with two copies of the gene encoding enzyme activity, CYP 2C19 ${ }^{*} 1 /{ }^{*} 1$, which is associated with the extensive metabolizer (normal) phenotype.

\section{Discussion:}

Severe gastrointestinal complications in renal transplant recipients include gastric or duodenal ulceration with subsequent bleeding. These complications are of unknown etiology and can occur in up to $39 \%$ of patient, but often are secondary to 
immunosuppressant medications such as mycophenolate or prednisone [7]. At many institutions, including our own, PPIs are used for ulcer prophylaxis. Therefore, most if not all renal transplant recipients taking PPIs for ulcer prophylaxis or treatment are at risk for the complication outlined in this report.

There are several sources of the potential interaction described in our patient. Of the five PPIs available in the United States, omeprazole is known to have the most drugdrug interactions [8]. Each PPI has a different reliance on CYP 2C19 and 3A and thus differential interaction potentials with tacrolimus. In the liver, CYP 2C19 is primarily involved with 5-hydroxylation of omeprazole with a minor contribution of CYP 3A [9]. However, once CYP 2C19 becomes saturated with high-dose omeprazole (40mg/day) in CYP 2C19 extensive metabolizers or in the case of CYP 2C19 poor metabolizers, CYP 3A becomes the dominant enzyme [10]. Additionally, an in vitro study suggested that at higher substrate concentrations, 5-hydroxylation and sulfoxidation of omeprazole are catalyzed principally by hepatic CYP 3A4 [11]. Although esomeprazole is the s-enantiomer of omeprazole, researchers have shown that CYP 2C19 plays a less predominant role in its metabolism [8, 12]. Esomeprazole inhibits intestinal PgP activity, and PgP inhibitors have been shown to increase oral bioavailability of tacrolimus [2]. Omeprazole also inhibits intestinal CYP 3A4 metabolism, another potential source of the interaction with tacrolimus [1]. By comparison, lansoprazole has not shown interactions due to intestinal PgP activity, and has been shown to be more reliant on CYP 2C19 metabolism than omeprazole [13]. Additionally, an in vitro study showed lansoprazole to have a lower CYP 3A4 inhibitory potential than omeprazole, suggesting less CYP 3A4 involvement in lansoprazole's metabolism [14].

While we considered the patient's concomitant antibiotics when investigating the cause of her tacrolimus toxicity, we considered them to be less likely sources for several reasons. Most importantly, the time course of the elevated levels and symptomatology did not fit with the starting and stopping of these drugs. Since the half-life of azithromycin is 68 hours, this would most likely not have caused the initial elevated levels found over two weeks after completing the course [15]. Furthermore, unlike other macrolides, azithromycin is not associated with CYP 450 drug interactions [16]. To our knowledge, only two case reports describe an interaction between tacrolimus and azithromycin, and these both showed toxicity within 24 hours of initiating the antibiotic $[17,18]$. The patient's courses of cefdinir continued throughout her presentation, so while we cannot rule out a complex interaction between PPIs, cefdinir and tacrolimus, we find it very unlikely such an interaction exists. Cefdinir is not known to affect CYP enzyme systems in humans, and to our knowledge, no case reports of cefdinir and tacrolimus interactions exist [19]. Finally, the cessation of the patient's omeprazole correlated directly with the relief of her toxicity symptoms as well as the marked decrease in tacrolimus concentrations. 
Except for one case report of a pediatric liver transplant recipient classified as a CYP 2C19 extensive metabolizer, the interaction between tacrolimus and PPIs has only been reported in subjects with CYP 2C19 polymorphisms [20]. One study concluded that the relative effect of CYP2C19 polymorphisms on the proton pump inhibitors is as follows: omeprazole $>$ pantoprazole $>$ lansoprazole $>$ rabeprazole [8]. CYP 2C19 poor metabolizers taking omeprazole $20 \mathrm{mg} /$ day had an increased concentration/dose ratio of tacrolimus, but those taking lansoprazole $30 \mathrm{mg} /$ day were not susceptible to the same interaction [21]. Rabeprazole has also been considered a safer treatment option than omeprazole in transplant patients receiving tacrolimus since it undergoes a mostly nonenzymatic metabolism with renal elimination of its metabolites [21]. Larger controlled studies are needed to determine the preferred PPI and starting dose for patients receiving tacrolimus. However, based on the pharmacokinetic properties of the drugs, along with the collective research and case reports, it may be prudent to avoid omeprazole and esomeprazole in patients receiving tacrolimus, regardless of CYP 2C19 genotype [10].

\section{Conclusion}

Until genetic variability is conclusively tied to interactions between tacrolimus and PPIs, careful monitoring of tacrolimus blood concentrations and toxicities should remain the standard of care. Prescribers should consider initial selection of a proton pump inhibitor carefully and avoid switching drug regimens once the patient is stabilized. If insurance coverage dictates a drug regimen change, once or twice weekly surveillance of tacrolimus levels should be instituted while the PPI is reaching steady state in the transplant patient. This case report highlights the importance of further studies to elucidate personalized medicine in patients with solid organ transplants requiring immunosuppressant medications.

\section{References}

1. Staatz CE, Tett SE (2004) Clinical pharmacokinetics and pharmacodynamics of tacrolimus in solid organ transplantation. Clin Pharmacokinet 43:623-653.

2. Christians U, Jacobsen W, Benet LZ, Lampen A (2002) Mechanisms of clinically relevant drug interactions associated with tacrolimus. Clin Pharmacokinet 41:813851.

3. Itagaki F, Homma M, Yuzawa K, Nishimura M, Naito S, Ueda N, Ohkohchi N, Kohda Y (2004) Effect of lansoprazole and rabeprazole on tacrolimus pharmacokinetics in healthy volunteers with CYP2C19 mutations. J Pharm Pharmacol 56:1055-1059. 
4. Hesselink DA, van Schaik RH, van der Heiden IP, van der Werf M, Gregoor PJ, Lindemans J, Weimar W, van Gelder T (2003) Genetic polymorphisms of the CYP3A4, CYP3A5, and MDR-1 genes and pharmacokinetics of the calcineurin inhibitors cyclosporine and tacrolimus. Clin Pharmacol Ther 74:245-254.

5. Katsakiori PF, Papapetrou EP, Goumenos DS, Nikiforidis GC, Flordellis CS (2010) Investigation of clinical interaction between omeprazole and tacrolimus in CYP3A5 non-expressors, renal transplant recipients. Ther Clin Risk Manag 6:265-269.

6. Tsuchiya N, Satoh S, Tada H, Li Z, Ohyama C, Sato K, Suzuki T, Habuchi T, Kato T (2004) Influence of CYP3A5 and MDR1 (ABCB1) polymorphisms on the pharmacokinetics of tacrolimus in renal transplant recipients. Transplantation 78:1182-1187.

7. Sarkio S, Halme L, Kyllonen L, Salmela K (2004) Severe gastrointestinal complications after 1,515 adult kidney transplantations. Transpl Int 17:505-510.

8. Ishizaki T, Horai Y (1999) Review article: cytochrome P450 and the metabolism of proton pump inhibitors--emphasis on rabeprazole. Aliment Pharmacol Ther 13 Suppl 3:27-36.

9. Andersson T, Miners JO, Veronese ME, Tassaneeyakul W, Meyer UA, Birkett DJ (1993) Identification of human liver cytochrome $\mathbf{P 4 5 0}$ isoforms mediating omeprazole metabolism. Br J Clin Pharmacol 36:521-530.

10. Rost KL, Roots I (1996) Nonlinear kinetics after high-dose omeprazole caused by saturation of genetically variable CYP2C19. Hepatology 23:1491-1497.

11. Yamazaki H, Inoue K, Shaw PM, Checovich WJ, Guengerich FP, Shimada T (1997) Different contributions of cytochrome P450 2C19 and 3A4 in the oxidation of omeprazole by human liver microsomes: effects of contents of these two forms in individual human samples. J Pharmacol Exp Ther 283:434-442.

12. Klotz U (2009) Impact of CYP2C19 polymorphisms on the clinical action of proton pump inhibitors (PPIs). Eur J Clin Pharmacol 65:1-2.

13. Shi S, Klotz U (2008) Proton pump inhibitors: an update of their clinical use and pharmacokinetics. Eur J Clin Pharmacol 64:935-951.

14. Li XQ, Andersson TB, Ahlstrom M, Weidolf L (2004) Comparison of inhibitory effects of the proton pump-inhibiting drugs omeprazole, esomeprazole, lansoprazole, pantoprazole, and rabeprazole on human cytochrome $\mathbf{P 4 5 0}$ activities. Drug Metab Dispos 32:821-827. 
15. Rapp RP (1998) Pharmacokinetics and pharmacodynamics of intravenous and oral azithromycin: enhanced tissue activity and minimal drug interactions. Ann Pharmacother 32:785-793.

16. Amacher DE, Schomaker SJ, Retsema JA (1991) Comparison of the effects of the new azalide antibiotic, azithromycin, and erythromycin estolate on rat liver cytochrome P-450. Antimicrob Agents Chemother 35:1186-1190.

17. Mori T, Aisa Y, Nakazato T, Yamazaki R, Ikeda Y, Okamoto S (2005) Tacrolimusazithromycin interaction in a recipient of allogeneic bone marrow transplantation. Transpl Int 18:757-758.

18. Shullo MA, Schonder K, Teuteberg JJ (2010) Elevated tacrolimus levels associated with intravenous azithromycin and ceftriaxone: a case report. Transplant Proc 42:1870-1872.

19. Niwa T, Shiraga T, Hashimoto T, Kagayama A (2004) Effect of cefixime and cefdinir, oral cephalosporins, on cytochrome $\mathbf{P 4 5 0}$ activities in human hepatic microsomes. Biol Pharm Bull 27:97-99.

20. Moreau C, Debray D, Loriot MA, Taburet AM, Furlan V (2006) Interaction between tacrolimus and omeprazole in a pediatric liver transplant recipient. Transplantation 81:487-488.

21. Hosohata K, Masuda S, Katsura T, Takada Y, Kaido T, Ogura Y, Oike F, Egawa H, Uemoto S, Inui K (2009) Impact of intestinal CYP2C19 genotypes on the interaction between tacrolimus and omeprazole, but not lansoprazole, in adult living-donor liver transplant patients. Drug Metab Dispos 37:821-826. 


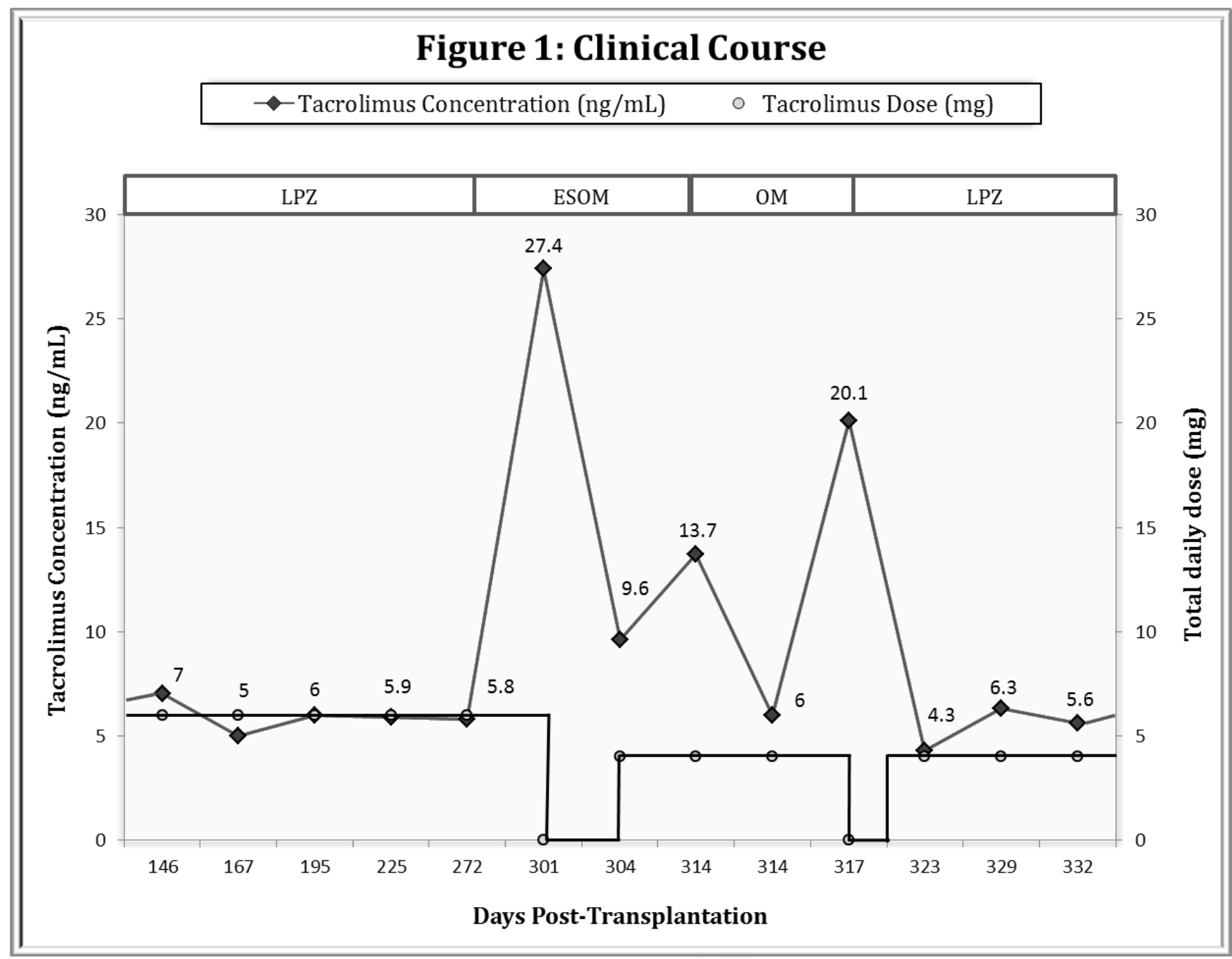

Figure 1 Legend: LPZ = Lansoprazole (30mg/day); ESOM = Esomeprazole (40mg/day); $\mathrm{OM}=$ Omeprazole $(20 \mathrm{mg} /$ day $)$

The official publication of the International Pediatric Transplant Association 


\section{Abstract:}

The shared hepatic cytochrome p450 (CYP) enzyme system between proton pump inhibitors (PPIs) and tacrolimus has been shown to cause clinically significant drug interactions, especially in patients who are classified as CYP 2C19 poor metabolizers. However, conflicting data suggests the mechanism needs to be further evaluated. A drug interaction between tacrolimus and omeprazole, esomeprazole, but not lansoprazole, occurred in an 18 year-old Caucasian female kidney transplant recipient classified as a CYP 2C19 extensive (normal) metabolizer. Until the relationship between genotype and this interaction is established definitively, prescribers should consider increased monitoring of tacrolimus blood concentrations when initiating or switching between PPIs.

\section{The shared metabolism of proton pump inhibitors (PPIs) and tacrolimus through} the cytochrome p450 (CYP) enzyme system has been associated with clinically significant drug interactions, especially in patients who are classified as CYP 2C19 poor metabolizers. However, existing data is conflicting, indicating that a single mechanism does not account for all interactions. A drug interaction between tacrolimus and omeprazole, esomeprazole, but not lansoprazole, occurred in an 18year-old female kidney transplant recipient classified as a CYP 2C19 extensive (normal) metabolizer. This case suggests further research is needed to establish the definitive mechanism of this potentially serious drug-drug interaction. Physicians prescribing PPIs in organ transplant recipients with tacrolimus immunosuppression should consider close pharmacokinetic monitoring of tacrolimus when starting or switching a PPI. 


\section{Background:}

Tacrolimus is a highly effective immunosuppressant drug used in combination to prevent solid organ transplant rejection. However, the narrow therapeutic window between under-dosing predisposing to increased risk of organ rejection and over-dosing leading to side effects such as acute and chronic nephrotoxicity, renal vasoconstriction and myelosuppression necessitates close monitoring of blood concentrations. Tacrolimus is absorbed through the intestinal multi-drug efflux transporter p-glycoprotein (PgP) and metabolized via hepatic CYP 3A enzyme systems, making it susceptible to many clinically significant drug interactions [1]. Most interactions are well known and managed through increased monitoring of tacrolimus blood concentrations, but few are as poorly elucidated interactions as with the proton pump inhibitors (PPIs).

Hepatic CYP 2C19 and 3A4 metabolize PPIs, and each PPI is metabolized differentially through the two pathways [2]. The shared hepatic CYP 3A4 system with tacrolimus is the presumed source of the interaction, but the exact pathway remains controversial. Many researchers have shown correlations between interaction potential of PPIs with tacrolimus and pharmacogenetic variability of CYP 2C19. CYP 2C19 genotypes classify patients as extensive metabolizers (EMs), intermediate metabolizers (IMs), or poor metabolizers (PMs) [3]. Poor metabolizers have markedly reduced activity of the 2C19 enzyme, leading to increased reliance on 3A4 for PPI metabolism [3]. Inhibition of tacrolimus clearance subsequently leads to supratherapeutic tacrolimus levels. However, conflicting data has spurred research into other pathways of the interaction, including intestinal PgP and hepatic CYP 3A5 genetic variability, all with more inconclusive or negative results [4-6].

In this case report, we present a renal transplant patient with a clinically relevant interaction between tacrolimus and esomeprazole, omeprazole, but not lansoprazole.

\section{Case Report:}

An 18-year-old Caucasian female with Wegener's Granulomatosis underwent a living donor renal transplant and placed on tacrolimus and mycophenolate for immunosuppression as well as lansoprazole for acid blockade. Concomitant chronic transplant medications included prednisone, sulfamethoxazole/trimethoprim, valganciclovir, and carvedilol. Tacrolimus blood levels were monitored routinely posttransplantation, with a goal range of trough concentrations between 5-8 ng/mL for maintenance immunosuppression. The patient maintained blood levels in this target range with $6 \mathrm{mg} /$ day of tacrolimus in two divided doses. Eight months post-transplant, lansoprazole (30mg/day) was switched to esomeprazole (40mg/day) due to insurance formulary changes. 
The patient presented one month later with fatigue and persistent sinusitis unresolved with courses of antibiotics. Tacrolimus blood levels were found to be elevated at $27.4 \mathrm{ng} / \mathrm{mL}$. The patient reported receiving a 10-day course of azithromycin from an outside physician, which she completed over 2 weeks prior to the first elevated tacrolimus trough, followed by a 10-day course of cefdinir completed 3 days prior to first elevated tacrolimus trough. The patient confirmed adherence to her tacrolimus regimen, and denied use of over-the-counter medications, grapefruit juice, and illicit drugs or alcohol. Her previous 9 months of tacrolimus levels were within the therapeutic window each time. Liver function tests of AST and ALT were within normal limits, and urine drug and toxicology screens were negative. The antibiotics were not considered likely causes of the elevated tacrolimus levels due to the time course of symptomatology and presentation in relation to antibiotic cessation. The tacrolimus dose was held for 24 hours and then reduced to $4 \mathrm{mg} /$ day. The blood levels were re-drawn the next morning and decreased to $9.6 \mathrm{ng} / \mathrm{mL}$.

Ten days later, the patient was admitted to the hospital with elevated serum creatinine and a tacrolimus level of $13.7 \mathrm{ng} / \mathrm{mL}$. She reported nausea and vomiting for several days, but reported no missed doses of tacrolimus. A second 21-day course of cefdinir had been prescribed prior to this presentation and was not considered as a causative agent due to the time course of symptomatology and lack of established evidence for an interaction with tacrolimus metabolism. Cefdinir was thus continued through and beyond the hospital admission. The tacrolimus was withdrawn and the patient was switched to omeprazole $40 \mathrm{mg} /$ day as per hospital formulary. Tacrolimus levels continued to remain elevated up to $20.1 \mathrm{ng} / \mathrm{mL}$. Figure 1 shows the course of tacrolimus blood concentrations and dosage during this time.

Physician and pharmacist review of the medication profile revealed a potential drug interaction between esomeprazole, omeprazole and tacrolimus. The patient was placed back on the lansoprazole. Tacrolimus levels normalized and symptoms of nausea and vomiting resolved. Within one week, the target range of $5-8 \mathrm{ng} / \mathrm{mL}$ was reached and tacrolimus titrated back up to the initial dose of $6 \mathrm{mg} /$ day. After obtaining written informed consent, a blood sample was sent to an outside facility for a cytochrome P450 2C19 genetic test done by polymerase chain reaction followed by DNA sequence analysis. The patients genotype came back with two copies of the gene encoding enzyme activity, CYP 2C19 ${ }^{*} 1 /{ }^{*} 1$, which is associated with the extensive metabolizer (normal) phenotype.

\section{Discussion:}

Severe gastrointestinal complications in renal transplant recipients include gastric or duodenal ulceration with subsequent bleeding. These complications are of unknown etiology and can occur in up to $39 \%$ of patient, but often are secondary to 
immunosuppressant medications such as mycophenolate or prednisone [7]. At many institutions, including our own, PPIs are used for ulcer prophylaxis. Therefore, most if not all renal transplant recipients taking PPIs for ulcer prophylaxis or treatment are at risk for the complication outlined in this report.

There are several sources of the potential interaction described in our patient. Of the five PPIs available in the United States, omeprazole is known to have the most drugdrug interactions [8]. Each PPI has a different reliance on CYP 2C19 and 3A and thus differential interaction potentials with tacrolimus. In the liver, CYP 2C19 is primarily involved with 5-hydroxylation of omeprazole with a minor contribution of CYP 3A [9]. However, once CYP 2C19 becomes saturated with high-dose omeprazole (40mg/day) in CYP 2C19 extensive metabolizers or in the case of CYP 2C19 poor metabolizers, CYP 3A becomes the dominant enzyme [10]. Additionally, an in vitro study suggested that at higher substrate concentrations, 5-hydroxylation and sulfoxidation of omeprazole are catalyzed principally by hepatic CYP 3A4 [11]. Although esomeprazole is the s-enantiomer of omeprazole, researchers have shown that CYP 2C19 plays a less predominant role in its metabolism [8, 12]. Esomeprazole inhibits intestinal PgP activity, and PgP inhibitors have been shown to increase oral bioavailability of tacrolimus [2]. Omeprazole also inhibits intestinal CYP 3A4 metabolism, another potential source of the interaction with tacrolimus [1]. By comparison, lansoprazole has not shown interactions due to intestinal PgP activity, and has been shown to be more reliant on CYP 2C19 metabolism than omeprazole [13]. Additionally, an in vitro study showed lansoprazole to have a lower CYP 3A4 inhibitory potential than omeprazole, suggesting less CYP 3A4 involvement in lansoprazole's metabolism [14].

While we considered the patient's concomitant antibiotics when investigating the cause of her tacrolimus toxicity, we considered them to be less likely sources for several reasons. Most importantly, the time course of the elevated levels and symptomatology did not fit with the starting and stopping of these drugs. Since the half-life of azithromycin is 68 hours, this would most likely not have caused the initial elevated levels found over two weeks after completing the course [15]. Furthermore, unlike other macrolides, azithromycin is not associated with CYP 450 drug interactions [16]. To our knowledge, only two case reports describe an interaction between tacrolimus and azithromycin, and these both showed toxicity within 24 hours of initiating the antibiotic $[17,18]$. The patient's courses of cefdinir continued throughout her presentation, so while we cannot rule out a complex interaction between PPIs, cefdinir and tacrolimus, we find it very unlikely such an interaction exists. Cefdinir is not known to affect CYP enzyme systems in humans, and to our knowledge, no case reports of cefdinir and tacrolimus interactions exist [19]. Finally, the cessation of the patient's omeprazole correlated directly with the relief of her toxicity symptoms as well as the marked decrease in tacrolimus concentrations. 
Except for one case report of a pediatric liver transplant recipient classified as a CYP 2C19 extensive metabolizer, the interaction between tacrolimus and PPIs has only been reported in subjects with CYP 2C19 polymorphisms [20]. One study concluded that the relative effect of CYP2C19 polymorphisms on the proton pump inhibitors is as follows: omeprazole $>$ pantoprazole $>$ lansoprazole $>$ rabeprazole [8]. CYP 2C19 poor metabolizers taking omeprazole $20 \mathrm{mg} /$ day had an increased concentration/dose ratio of tacrolimus, but those taking lansoprazole $30 \mathrm{mg} /$ day were not susceptible to the same interaction [21]. Rabeprazole has also been considered a safer treatment option than omeprazole in transplant patients receiving tacrolimus since it undergoes a mostly nonenzymatic metabolism with renal elimination of its metabolites [21]. Larger controlled studies are needed to determine the preferred PPI and starting dose for patients receiving tacrolimus. However, based on the pharmacokinetic properties of the drugs, along with the collective research and case reports, it may be prudent to avoid omeprazole and esomeprazole in patients receiving tacrolimus, regardless of CYP 2C19 genotype [10].

\section{Conclusion}

Until genetic variability is conclusively tied to interactions between tacrolimus and PPIs, careful monitoring of tacrolimus blood concentrations and toxicities should remain the standard of care. Prescribers should consider initial selection of a proton pump inhibitor carefully and avoid switching drug regimens once the patient is stabilized. If insurance coverage dictates a drug regimen change, once or twice weekly surveillance of tacrolimus levels should be instituted while the PPI is reaching steady state in the transplant patient. This case report highlights the importance of further studies to elucidate personalized medicine in patients with solid organ transplants requiring immunosuppressant medications.

\section{References}

1. Staatz CE, Tett SE (2004) Clinical pharmacokinetics and pharmacodynamics of tacrolimus in solid organ transplantation. Clin Pharmacokinet 43:623-653.

2. Christians U, Jacobsen W, Benet LZ, Lampen A (2002) Mechanisms of clinically relevant drug interactions associated with tacrolimus. Clin Pharmacokinet 41:813851.

3. Itagaki F, Homma M, Yuzawa K, Nishimura M, Naito S, Ueda N, Ohkohchi N, Kohda Y (2004) Effect of lansoprazole and rabeprazole on tacrolimus pharmacokinetics in healthy volunteers with CYP2C19 mutations. J Pharm Pharmacol 56:1055-1059. 
4. Hesselink DA, van Schaik RH, van der Heiden IP, van der Werf M, Gregoor PJ, Lindemans J, Weimar W, van Gelder T (2003) Genetic polymorphisms of the CYP3A4, CYP3A5, and MDR-1 genes and pharmacokinetics of the calcineurin inhibitors cyclosporine and tacrolimus. Clin Pharmacol Ther 74:245-254.

5. Katsakiori PF, Papapetrou EP, Goumenos DS, Nikiforidis GC, Flordellis CS (2010) Investigation of clinical interaction between omeprazole and tacrolimus in CYP3A5 non-expressors, renal transplant recipients. Ther Clin Risk Manag 6:265-269.

6. Tsuchiya N, Satoh S, Tada H, Li Z, Ohyama C, Sato K, Suzuki T, Habuchi T, Kato T (2004) Influence of CYP3A5 and MDR1 (ABCB1) polymorphisms on the pharmacokinetics of tacrolimus in renal transplant recipients. Transplantation 78:1182-1187.

7. Sarkio S, Halme L, Kyllonen L, Salmela K (2004) Severe gastrointestinal complications after 1,515 adult kidney transplantations. Transpl Int 17:505-510.

8. Ishizaki T, Horai Y (1999) Review article: cytochrome P450 and the metabolism of proton pump inhibitors--emphasis on rabeprazole. Aliment Pharmacol Ther 13 Suppl 3:27-36.

9. Andersson T, Miners JO, Veronese ME, Tassaneeyakul W, Meyer UA, Birkett DJ (1993) Identification of human liver cytochrome $\mathbf{P 4 5 0}$ isoforms mediating omeprazole metabolism. Br J Clin Pharmacol 36:521-530.

10. Rost KL, Roots I (1996) Nonlinear kinetics after high-dose omeprazole caused by saturation of genetically variable CYP2C19. Hepatology 23:1491-1497.

11. Yamazaki H, Inoue K, Shaw PM, Checovich WJ, Guengerich FP, Shimada T (1997) Different contributions of cytochrome P450 2C19 and 3A4 in the oxidation of omeprazole by human liver microsomes: effects of contents of these two forms in individual human samples. J Pharmacol Exp Ther 283:434-442.

12. Klotz U (2009) Impact of CYP2C19 polymorphisms on the clinical action of proton pump inhibitors (PPIs). Eur J Clin Pharmacol 65:1-2.

13. Shi S, Klotz U (2008) Proton pump inhibitors: an update of their clinical use and pharmacokinetics. Eur J Clin Pharmacol 64:935-951.

14. Li XQ, Andersson TB, Ahlstrom M, Weidolf L (2004) Comparison of inhibitory effects of the proton pump-inhibiting drugs omeprazole, esomeprazole, lansoprazole, pantoprazole, and rabeprazole on human cytochrome $\mathbf{P 4 5 0}$ activities. Drug Metab Dispos 32:821-827. 
15. Rapp RP (1998) Pharmacokinetics and pharmacodynamics of intravenous and oral azithromycin: enhanced tissue activity and minimal drug interactions. Ann Pharmacother 32:785-793.

16. Amacher DE, Schomaker SJ, Retsema JA (1991) Comparison of the effects of the new azalide antibiotic, azithromycin, and erythromycin estolate on rat liver cytochrome P-450. Antimicrob Agents Chemother 35:1186-1190.

17. Mori T, Aisa Y, Nakazato T, Yamazaki R, Ikeda Y, Okamoto S (2005) Tacrolimusazithromycin interaction in a recipient of allogeneic bone marrow transplantation. Transpl Int 18:757-758.

18. Shullo MA, Schonder K, Teuteberg JJ (2010) Elevated tacrolimus levels associated with intravenous azithromycin and ceftriaxone: a case report. Transplant Proc 42:1870-1872.

19. Niwa T, Shiraga T, Hashimoto T, Kagayama A (2004) Effect of cefixime and cefdinir, oral cephalosporins, on cytochrome $\mathbf{P 4 5 0}$ activities in human hepatic microsomes. Biol Pharm Bull 27:97-99.

20. Moreau C, Debray D, Loriot MA, Taburet AM, Furlan V (2006) Interaction between tacrolimus and omeprazole in a pediatric liver transplant recipient. Transplantation 81:487-488.

21. Hosohata K, Masuda S, Katsura T, Takada Y, Kaido T, Ogura Y, Oike F, Egawa H, Uemoto S, Inui K (2009) Impact of intestinal CYP2C19 genotypes on the interaction between tacrolimus and omeprazole, but not lansoprazole, in adult living-donor liver transplant patients. Drug Metab Dispos 37:821-826. 